\title{
Photoluminescence dynamics due to biexcitons and exciton-exciton scattering in the layered-type semiconductor $\mathrm{PbI}_{2}$
}

\author{
M. Ando, ${ }^{1}$ M. Yazaki, ${ }^{1}$ I. Katayama, ${ }^{1}$ H. Ichida, ${ }^{2}$ S. Wakaiki, ${ }^{3}$ Y. Kanematsu, ${ }^{2,3}$ and J. Takeda ${ }^{1}$ \\ ${ }^{1}$ Department of Physics, Graduate School of Engineering, Yokohama National University, Yokohama 240-8501, Japan \\ ${ }^{2}$ Venture Business Laboratory, Osaka University, Suita 565-0871, Japan \\ ${ }^{3}$ Department of Material and Life Science, Graduate School of Engineering, Osaka University, Suita 565-0871, Japan
}

(Received 11 April 2012; revised manuscript received 13 September 2012; published 8 October 2012)

\begin{abstract}
The dynamics of photoluminescence due to biexcitons and exciton-exciton scattering $(M$ and $P$ emissions, respectively) has been investigated in the layered-type semiconductor $\mathrm{PbI}_{2}$ by using the optical Kerr gate method. We simultaneously observed $P$ and $M$ emissions under high-density excitation. The $M$ emission emerges instantaneously, whereas the $P$ emission shows a delayed onset whose latency increases as the excitation photon energy increases. The latency to onset indicates that the $P$ emission takes place after the relaxation of excitons with excess energy toward the bottleneck region via exciton-longitudinal optical (LO) phonon scattering processes. Based on the time-dependent peak energy shift of the $P$ emission and a line-shape analysis of the $M$ emission, we evaluated the effective temperatures of both photogenerated excitonic and biexcitonic systems as well as the self-energy due to the collisions among biexcitons. We conclude that these systems are separately formed in space owing to potential fluctuations between $\mathrm{PbI}_{2}$ layers, and independently reach thermal equilibrium after $\sim 30 \mathrm{ps}$ with different cooling processes. The exciton-exciton and exciton-LO phonon scattering processes play an important role in cooling the excitonic system, whereas the biexciton-biexciton and biexciton-exciton collisions are dominant in cooling the biexcitonic system.
\end{abstract}

DOI: 10.1103/PhysRevB.86.155206

PACS number(s): 78.47.jd, 78.20.-e, 78.55.-m

\section{INTRODUCTION}

The photoluminescence (PL) properties of high-density excitons in semiconductors have attracted a great deal of attention in the fields of fundamental semiconductor physics and device applications. Generally, as the excitation photon density increases, PL due to biexcitons (so-called $M$ emission) first appears. Subsequently, after the saturation of $M$ emission, PL caused by inelastic scattering of excitons (so-called $P$ emission) emerges in place of $M$ emission. In the case of $M$ emission, one of two excitons in a biexcitonic state is radiatively annihilated, leaving the other as a free exciton. In contrast, in the case of $P$ emission, the inelastic scattering between two excitons in the excitonic state $(n=1)$ excites one into a higher excitonic $(n \geqslant 2)$ or continuum state $(n=\infty)$, and scatters the other into the lower polariton branch. Recently, owing to the invention of ultrafast laser spectroscopy, the PL dynamics of $M$ and $P$ emissions have been extensively studied in wide-gap semiconductors. ${ }^{1-5}$ For example, the formation and decay processes of biexcitons and laser emission originated from a biexcitonic state were clarified in $\mathrm{CuCl}$ quantum dots, ${ }^{3}$ while the dynamics of exciton-exciton and exciton-phonon scattering processes were revealed in $\mathrm{CuI}^{4}$ and $\mathrm{ZnO}{ }^{5}$ However, although both $M$ and $P$ emissions relate to two excitons, whether the excitons are bound to form a biexciton or cause mutual scattering is still unclear. Furthermore, the coexistence dynamics between excitonic and biexcitonic systems have not been well understood to date.

The layered-type semiconductor $\mathrm{PbI}_{2}$ has a direct band gap of $\sim 2.54 \mathrm{eV}$ and an exciton binding energy of $30 \mathrm{meV}^{6}$ The optical properties of the $M$ emission are well known under band-to-band excitation, ${ }^{7,8}$ while very recently the $P$ emission was observed via a two-photon absorption process for the band-to-band transition. In the two-photon absorption process, formation of biexcitons could be radically suppressed making it possible to observe the $P$ emission. ${ }^{9}$ Given that both the $M$ and $P$ emissions are clearly observed, $\mathrm{PbI}_{2}$ is a suitable material to investigate the PL dynamics between the $M$ and $P$ emissions, both of which are related to two excitons.

In the present work, we investigated the PL dynamics due to biexcitons and exciton-exciton scattering processes in the layered-type semiconductor $\mathrm{PbI}_{2}$ under high-density excitations. Using the femtosecond (fs) optical Kerr gate (OKG) method, we successfully measured ultrafast dynamics of $M$ and $P$ emissions under band-to-band excitations. As a result, we evaluated the full time-dependent behaviors of these emissions such as the spectral shift and effective temperature. From the results, we found that in addition to exciton-exciton collision, the exciton-longitudinal optical (LO) phonon scattering process plays an important role in the $P$ emission dynamics, whereas the biexciton-biexciton and biexciton-exciton collision processes are dominant for the $M$ emission dynamics. We also speculate that the excitonic and biexcitonic systems are separately formed in space owing to the existence of potential fluctuations between the $\mathrm{PbI}_{2}$ layers, and independently reach thermal equilibrium after $\sim 30 \mathrm{ps}$ via different cooling processes.

\section{EXPERIMENTAL DETAILS}

Plateletlike $4 \mathrm{H}-\mathrm{PbI}_{2}$ single crystals were grown under vapor phase as previously reported. ${ }^{10}$ The typical thickness of samples is $10-100 \mu \mathrm{m}$. The sample was kept at $20 \mathrm{~K}$ in a temperature-controlled cryostat.

We used a Ti:sapphire regenerative amplifier laser system with a center wavelength of $800 \mathrm{~nm}$, repetition rate of $1 \mathrm{kHz}$, and pulse duration of $150 \mathrm{fs}$ as the light source. The excitation light was the second harmonic pulse $(400 \mathrm{~nm} / 3.1 \mathrm{eV})$, whose energy corresponds to the band-to-band transition of $\mathrm{PbI}_{2}$. 
When tuning the excitation photon energy from the bandto-band excitation energy to exciton resonance excitation energy, we used a home-built optical parametric amplifier (OPA) pumped with a Ti:sapphire regenerative amplifier. Time-resolved PL spectra were measured by the OKG method, whose temporal resolution is $1.2 \mathrm{ps}$, with $\mathrm{CS}_{2}$ solution as a Kerr medium. ${ }^{11}$ The time-integrated and time-resolved PL spectra were detected by a monochromator with a charge coupled device (CCD) detector cooled at liquid nitrogen temperature. The experimental setup is almost the same as that reported in Ref. 12.

\section{RESULTS AND DISCUSSION}

Figure 1(a) shows time-integrated PL spectra under various excitation photon densities at $20 \mathrm{~K}$. Under weak excitation density, the $M$ and $L$ emission bands were observed around 2.50 and $2.44 \mathrm{eV}$, respectively. The $M$ emission comes from the radiative annihilation of biexcitons, ${ }^{7,8}$ whereas the $L$ emission is due to the localized excitons that are trapped by potential fluctuations. ${ }^{13}$ Because an intense regenerative amplifier system was used as an excitation light source, the high-density PL band due to biexcitons was observed instead of the PL bands due to free and bound excitons even under the weak excitation limit of $0.04 \mathrm{~mJ} / \mathrm{cm}^{2}$. On the other hand, the excitation density of $0.74 \mathrm{~mJ} / \mathrm{cm}^{2}$ yields the exciton density of $\sim 5.0 \times 10^{18} \mathrm{~cm}^{-3}$, whose value is below the Mott transition density expected in this material. Therefore, the PL band due to electron-hole plasma should not be observed under our experimental conditions. The PL intensities of the $L$ and $M$ emission bands increase linearly and quadratically, respectively, and then saturate with increasing the excitation density, as shown in Fig. 1(b). The spectral shape of the $M$ emission is asymmetric; the tail toward the lower-energy side reflects the Boltzmann distribution of the biexcitonic system with an effective temperature, while that of the higher-energy

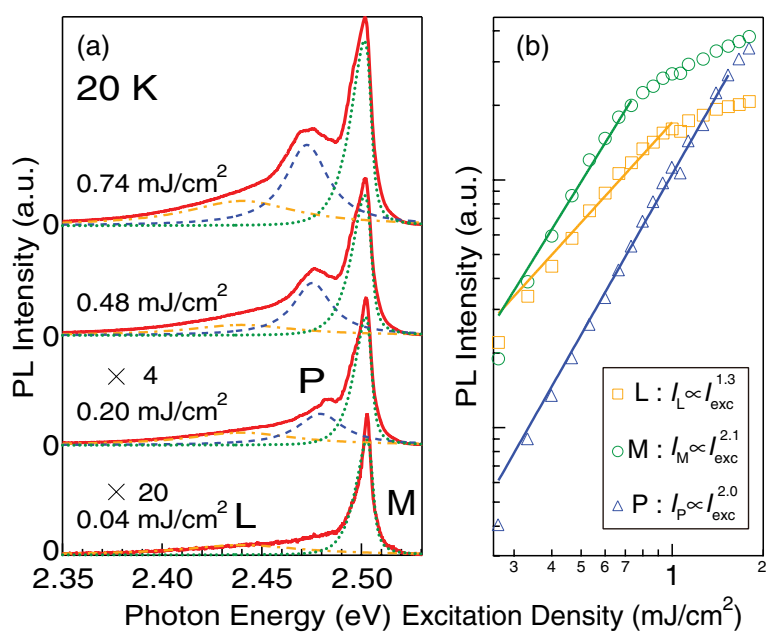

FIG. 1. (Color online) (a) Photoluminescence (PL) spectra under various excitation photon densities at $20 \mathrm{~K} . M, P$, and $L$ denote the emission bands due to biexcitons, exciton-exciton scattering, and excitons localized by potential fluctuations, respectively. (b) Excitation photon density dependence of the PL intensities for the $M, P$, and $L$ emissions. side originates from the biexciton-biexciton collisions due to the intense pump power. ${ }^{14}$

As the excitation density increases, a new emission band ( $P$ emission band) with a nearly Lorentzian shape emerges around $2.47 \mathrm{eV}$; its intensity increases quadratically with increasing excitation photon density without a saturation behavior. The peak energy of the observed $P$ emission is close to the peak energies of the steady-state $P_{\infty}$ and $P_{2}$ emissions expected in $\mathrm{PbI}_{2}$. Here, the energy position of the $P_{\infty}$ and $P_{2}$ emissions is given by

$$
\hbar \omega_{P}=E_{n=1}-\left(E_{n=2, \infty}-E_{n=1}\right)-\frac{3}{2} k_{\mathrm{B}} T,
$$

where $E_{n=1}, E_{n=2}$, and $E_{n=\infty}$ are the energies of the $n=1$ excitonic, $n=2$ excitonic, and continuum states, respectively, $k_{\mathrm{B}}$ is the Boltzmann factor and $T$ is the effective temperature of the excitonic system. ${ }^{15}$ By inserting the excitonic energies of $\mathrm{PbI}_{2}\left(E_{n=1}=2.506, E_{n=2}=2.529\right.$, and $E_{n=\infty}=2.536 \mathrm{eV}$, respectively) and the lattice temperature $(20 \mathrm{~K})$ as $T$ into Eq. (1), the energy positions of the $P_{\infty}$ and $P_{2}$ emissions are expected to be 2.476 and $2.483 \mathrm{eV}$, respectively. The effective temperature of the excitonic system should be higher than the lattice temperature and increases with increasing the excitation photon density. Therefore, the energy position of the observed $P$ emission is lower than the energy positions of the estimated $P_{\infty}$ and $P_{2}$ emissions, and decreases with increasing excitation photon density, as shown in Fig. 1(a). Judging from these experimental results, the observed $P$ emission band most likely originates from the inelastic exciton-exciton scattering process.

Figures 2(a) and 2(c) show the two-dimensional timeenergy images of the PL at $20 \mathrm{~K}$ under the excitation densities of 0.74 and $0.15 \mathrm{~mJ} / \mathrm{cm}^{2}$, respectively. The corresponding time-resolved PL spectra with different time delays are also
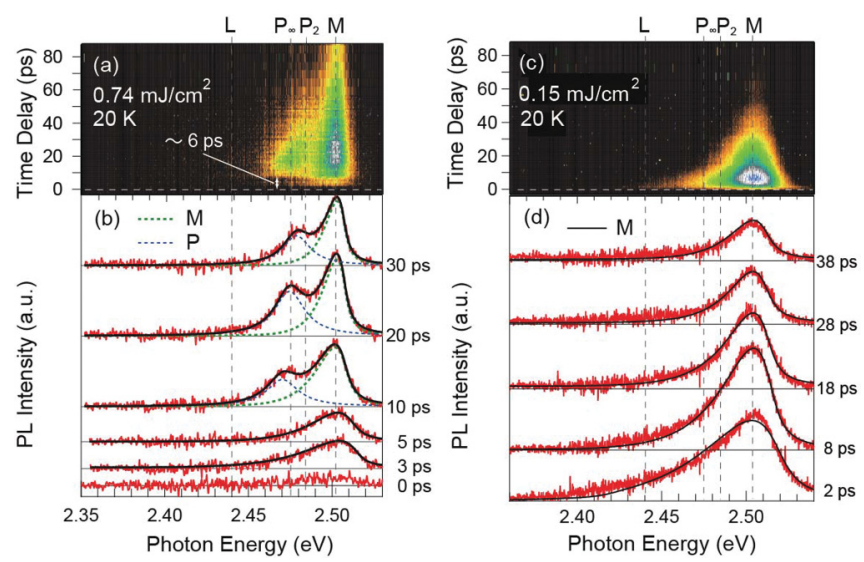

FIG. 2. (Color online) (a) Time-energy two-dimensional image of PL measured by the optical Kerr gate method and (b) time-resolved PL spectra with different time delays of $0,3,5,10,20$, and 30 ps under an excitation density of $0.74 \mathrm{~mJ} / \mathrm{cm}^{2}$. (c) Time-energy two-dimensional image of PL and (d) time-resolved PL spectra with different time delays of $2,8,18,28$, and 38 ps under an excitation density of $0.15 \mathrm{~mJ} / \mathrm{cm}^{2}$. The dashed (blue) and dotted (green) curves show the $P$ and $M$ emission bands, respectively. The solid (black) curves show the total emission bands. The vertical dashed lines in (a), (b) and (c), (d) show the peak energy positions of the steady-state $L, P_{\infty}, P_{2}$, and $M$ emission bands, respectively, expected in $\mathrm{PbI}_{2}$. 
shown in Figs. 2(b) and 2(d). The vertical dashed lines show the peak energy positions of the steady-state $L, P_{\infty}, P_{2}$, and $M$ emissions, respectively, which are expected in $\mathrm{PbI}_{2}$. The $L$ emission could not be observed by the time-resolved PL measurements because the lifetime of the $L$ emission band $(\geqslant \sim 1 \mathrm{~ns})$ is much longer than the time window of our experiment $(\sim 1.2 \mathrm{ps}) .^{13}$

As shown in Fig. 2, only the $M$ emission band is observed under the weak excitation density, whereas the $M$ and $P$ emission bands are observed together under the high-density excitation. To analyze dynamical behaviors of the $M$ and $P$ emission bands separately, we performed a line-shape analysis. The $M$ emission was analyzed by taking account of the thermal distribution of the biexcitons and the biexciton-biexciton collision process as follows: ${ }^{14}$

$$
I(E) \propto \int_{0}^{\infty} \frac{n(\varepsilon, T) \Gamma \varepsilon^{1 / 2} d \varepsilon}{(E-\Sigma-\varepsilon)^{2}+\Gamma^{2}} .
$$

Here, $I(E)$ is the $M$ emission intensity as a function of biexciton energy $E$, and $n(\varepsilon, T)=\exp \left(-\varepsilon / k_{\mathrm{B}} T\right)$ is the Boltzmann distribution function with an effective temperature. $\Gamma$ and $\Sigma$ originate from self-energy due to the collision among biexcitons; $\Sigma$ corresponds to the energy gain due to the collision and affects the peak shift of the biexciton, whereas $\Gamma$ contributes to the actual relaxation process and participates in broadening the spectral width. The Boltzmann distribution with a given effective temperature modifies the low-energy tail of the $M$ emission band. Therefore, we could independently determine the three adjustable parameters, $\Gamma, \Sigma$, and $T$, at given time delays by our line-shape analysis. On the other hand, the $P$ emission was phenomenologically fitted with a Lorentzian function. The dotted and broken lines in Fig. 2(b) show the $M$ and $P$ emission bands, respectively, whereas solid lines in Figs. 2(b) and 2(d) represent the total emission line shape. As seen in Figs. 2(b) and 2(d), we could reproduce all of the observed data well by this procedure. Therefore, we can evaluate the time-dependent dynamical behaviors of the $M$ and $P$ emissions in full detail.

The $M$ emission emerges immediately after the irradiation of the excitation pulse, whereas the $P$ emission appears with a time delay, as shown in Figs. 2(a) and 2(c). Figure 3 shows the spectral-integrated PL intensities of the $M$ and $P$

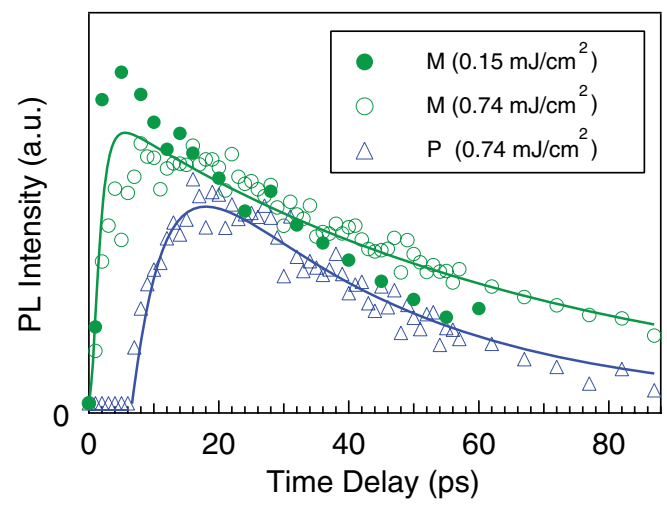

FIG. 3. (Color online) Time evolutions of the integrated $P$ and $M$ emission bands. The solid lines show the best fits calculated by the method described in the main text. emissions as a function of time delay. The $M$ emission emerges instantaneously within the temporal resolution of the system response function, whereas the $P$ emission has an onset time to appear. Therefore, the time evolution of the $M$ and $P$ emission bands $I(t)$ was phenomenologically fitted with the following function $g(t)$ convoluted with the system response function $f(t)$; that is,

$$
I(t) \propto \int_{-\infty}^{t} f(t-\tau) g(\tau) d \tau
$$

with

$$
g(t)=\left\{1-\exp \left(-\frac{t-\tau_{\text {onset }}}{\tau_{\mathrm{r}}}\right)\right\} \exp \left(-\frac{t-\tau_{\text {onset }}}{\tau_{\mathrm{d}}}\right),
$$

where $\tau_{\mathrm{r}}, \tau_{\mathrm{d}}$, and $\tau_{\text {onset }}$ are the rise, decay, and onset times, respectively. For example, under the excitation density of $0.74 \mathrm{~mJ} / \mathrm{cm}^{2}$, we found that the $M$ emission emerges instantaneously within the temporal resolution of the system response function and decays with $\tau_{\mathrm{d}}=76 \mathrm{ps}$. In contrast, the $P$ emission has onset and decay times of 6.0 and $37 \mathrm{ps,}$ respectively. The obtained decay times contain information on various physical parameters such as the self-energy and the effective temperature. Therefore, the time-dependent behavior of each physical parameter, which comes from the line-shape analysis, will be individually discussed in the following paragraphs.

Figure 4(a) shows the time evolution of the peak energies of the $M$ and $P$ emission bands under the excitation density of $0.74 \mathrm{~mJ} / \mathrm{cm}^{2}$. The dashed lines show the expected peak energy positions of the $M, P_{2}$, and $P_{\infty}$ emission bands, respectively. The peak energy of the $M$ emission shifts to the lower-energy side and approaches the energy expected in the steady state. In addition, as seen in Fig. 4(b), the magnitude of real and imaginary parts of the self-energy estimated from the lineshape analysis decreases as a function of time along a curve with two exponential decay components, the decay times of which were 4.5 and $32 \mathrm{ps}$ in $\Gamma$, and 4.9 and $39 \mathrm{ps}$ in $\Sigma$. This is because the probability of the collisions in a biexcitonic system decreases as the biexciton density decreases with time through various decay processes. ${ }^{16}$ In Ref. 14 , the line shape of the $M$ emission in $\mathrm{CdS}$ and $\mathrm{CdSe}$ samples was analyzed by taking account of the broadening due to the collisions. However, the effect of $\Sigma$ on the spectral shape could not be detected because of the smaller value of $\Sigma$ in comparison with that of $k_{\mathrm{B}} T .{ }^{14} \mathrm{In}$ our case, on the other hand, intense femtosecond laser pulses induce the effective collisions among high-density biexcitons, leading to first observation of the clear temporal change of $\Sigma$ as well as $\Gamma$.

To confirm the collision process among biexcitons, we also evaluated the time-dependent self-energy under the weak excitation density of $0.15 \mathrm{~mJ} / \mathrm{cm}^{2}$ using the same analytical procedure. As shown in Fig. 4(c), $\Sigma$ decreases biexponentially with time having longer decay times of 7.0 and $\geqslant \sim 50$ ps. Using the same time constants, the temporal behavior of $\Gamma$ could be also reproduced [see broken lines in Fig. 4(c)]. The magnitude of $\Sigma$ in the whole temporal region and that of $\Gamma$ at the early stage under the weak excitation density $\left(0.15 \mathrm{~mJ} / \mathrm{cm}^{2}\right)$ are smaller than those under the high excitation density $\left(0.74 \mathrm{~mJ} / \mathrm{cm}^{2}\right)$. The biexciton density photogenerated under the weak excitation density is lower than that under 


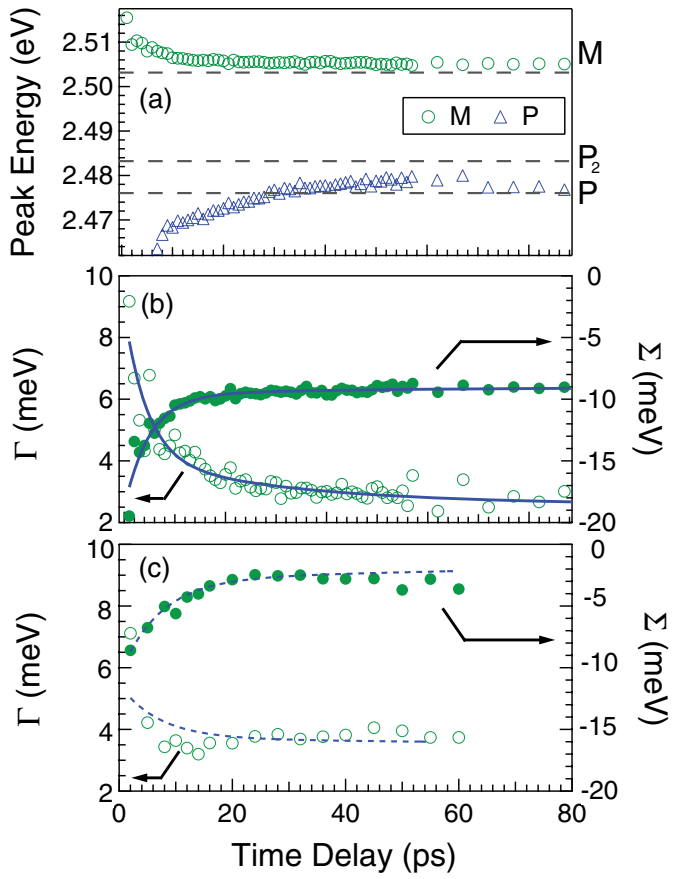

FIG. 4. (Color online) (a) Peak energy shift of the $M$ and $P$ emission bands as a function of time delay under an excitation density of $0.74 \mathrm{~mJ} / \mathrm{cm}^{2}$. The dashed lines show the expected peak energy positions of the $M, P_{2}$, and $P_{\infty}$ bands, respectively. (b) Time evolution of real and imaginary parts of the self-energy, $\Gamma$ and $\Sigma$, under an excitation density of $0.74 \mathrm{~mJ} / \mathrm{cm}^{2}$ estimated from the line-shape analysis (see main text). (c) Time evolution of $\Gamma$ and $\Sigma$ under an excitation density of $0.15 \mathrm{~mJ} / \mathrm{cm}^{2}$. The solid and dashed curves are fitted with two exponential functions.

the high excitation density, leading to less probability of the collisions in a biexcitonic system. As a result, the decay times become slower and the magnitude of the self-energy decreases with decreasing the excitation density. In contrast, the value of $\Gamma$ after a long time delay (3-4 meV) seems to be independent of the excitation density. The imaginary part of the self-energy, $\Gamma$, is directly connected to the actual relaxation process. After the collisions among biexcitons are almost completed, the relaxation might be governed by nonradiative processes such as trapping into impurities and potential fluctuations, which are independent of the biexciton density.

In contrast to the $M$ emission, the peak energy of the $P$ emission shifts to higher energy after an onset time of $6.0 \mathrm{ps,}$ and then approaches that of the steady-state $P_{\infty}$ emission [see Fig. 4(a)]. As shown in Eq. (1), the energy of the $P$ emission depends on the effective temperature. Therefore, the higherenergy shift of the $P$ emission is attributed to a reduction of the effective temperature of the excitonic system in time. In the following, we focus on the coexistence dynamics between excitonic and biexcitonic systems.

Figure 5 shows the effective temperatures of the excitonic and biexcitonic systems under the excitation density of $0.74 \mathrm{~mJ} / \mathrm{cm}^{2}$ as a function of time delay. Here, the effective temperature of the excitonic system is estimated from the energy difference between $E_{n=\infty}$ and $E_{n=1}$ by using Eq. (1), whereas that of the biexcitonic system is obtained from the line-shape analysis of the $M$ emission. The effective

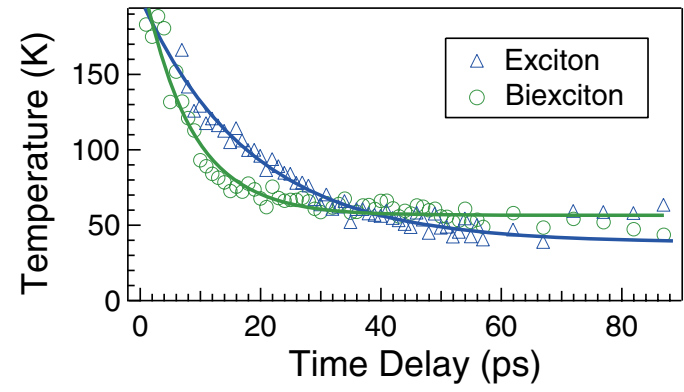

FIG. 5. (Color online) Time evolution of effective temperatures of the excitonic and biexcitonic systems; the former was estimated from the peak energy of the $P$ emission, whereas the latter was estimated from the spectral shape analysis for the $M$ emission. The solid curves are the best fits calculated with exponential functions (see main text).

temperatures in the excitonic and biexcitonic systems both decrease with time from an initial effective temperature of $\sim 200 \mathrm{~K}$ to nearly the lattice temperature of $30-50 \mathrm{~K}$ with different decay profiles. The effective temperature of the excitonic system declines according a single exponential decay with a time constant of $15 \mathrm{ps}$; this decline may reflect the relaxation due to the exciton-exciton collisions. In contrast, the effective temperature of the biexcitonic system decreases along a curve with two exponential decay components, whose time constants ( 7.1 and $39 \mathrm{ps)}$ are close to the fast and slow decay times estimated from the time-dependent selfenergies [see Fig. 4(b)]. Therefore, the fast decay component comes from the collision among biexcitons, whereas the slow component may come from exciton-biexciton collision and/or nonradiative pathways into impurities and potential fluctuations after the biexciton-biexciton collision process is almost completed. These results strongly suggest that the excitonic and biexcitonic systems generated by the same pump laser pulses have different cooling processes and are thermalized separately from each other. We will discuss these results in the last section of the paper.

To reveal the origin of the onset time that was observed only in the $P$ emission, we measured the excitation photon energy dependence of the PL by using an OPA pumped by a regenerative Ti:sapphire amplifier system. Figure 6 shows the onset time of the $P$ emission as a function of the excitation photon energy at $20 \mathrm{~K}$. The observed onset time of the $P$

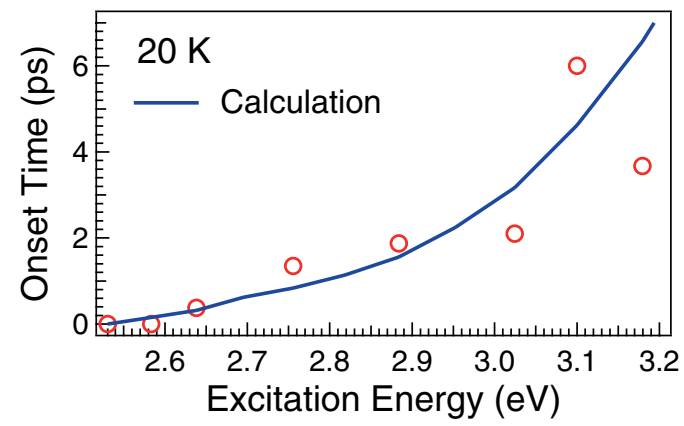

FIG. 6. (Color online) The onset time of the $P$ emission as a function of excitation photon energy at $20 \mathrm{~K}$. The solid (blue) line shows the calculated onset time based on the exciton-LO phonon scattering process. 
emission increases with the excitation photon energy owing to the following cascade relaxation process. Under band-toband excitation, the photogenerated exciton with an excess energy relaxes to the bottleneck region along the excitonpolariton dispersion curve by emitting multiple LO phonons via the exciton-LO phonon scattering process. Subsequently, at the bottleneck region, the $P$ emission effectively occurs via the exciton-exciton scattering process, leading to the delayed onset. In contrast, under the exciton resonance excitation, the $P$ emission occurs immediately via exciton-exciton scattering because excitons are directly generated around the bottleneck region. We quantitatively calculated the onset time of the $P$ emission by the same procedure as that reported in Ref. 17 . In this calculation, we used $\Omega=13.9 \mathrm{meV}^{18} \varepsilon_{\infty}=6.1$, $\varepsilon_{0}=26.4,{ }^{19} a_{\mathrm{ex}}=1.9 \mathrm{~nm},{ }^{20} m_{\mathrm{e}}=0.48 m_{0}, m_{\mathrm{h}}=0.20 m_{0},{ }^{21}$ and $E_{n=1}=2.506 \mathrm{eV}$ as physical parameters, where $\Omega$ is the LO-phonon energy, $\varepsilon_{\infty}$ the dielectric constant at infinite frequency, $\varepsilon_{0}$ the static dielectric constant, $a_{\mathrm{ex}}$ the exciton Bohr radius, $m_{\mathrm{e}}$ the electron mass, $m_{\mathrm{h}}$ the hole mass, and $E_{n=1}$ the exciton energy at state $n=1$. The calculated result is in quite good agreement with the experimental data, as shown by the solid line in Fig. 5. In contrast to the $P$ emission, the $M$ emission does not show any onset time to emerge as shown in Fig. 2. The formation and decay dynamics of biexcitons have been extensively studied in wide-gap semiconductors such as $\mathrm{CuCl}$, and the $M$ emission generally appears without any time delay. ${ }^{22,23}$ The biexcitonic state can be produced via the two formation processes: resonant two-photon absorption for the biexcitonic state and exciton-exciton interaction to form a biexciton. In the former process, the biexcitons are directly and effectively generated because of the resonant transition to an intermediate excitonic state and the giant oscillator strength from the excitonic to biexcitonic states. ${ }^{9,24}$ Because this process might be dominant, the $M$ emission emerges instantaneously within the temporal resolution of the system response function as shown in Figs. 2 and 3.

Finally, we discuss why the excitonic and biexcitonic systems are independently thermalized with different cooling processes. One possibility is that the excitonic and biexcitonic systems are separately formed in space. To test this conjecture, we measured the time-integrated PL spectra at various areas of the $\mathrm{PbI}_{2}$ sample with the same excitation intensity of

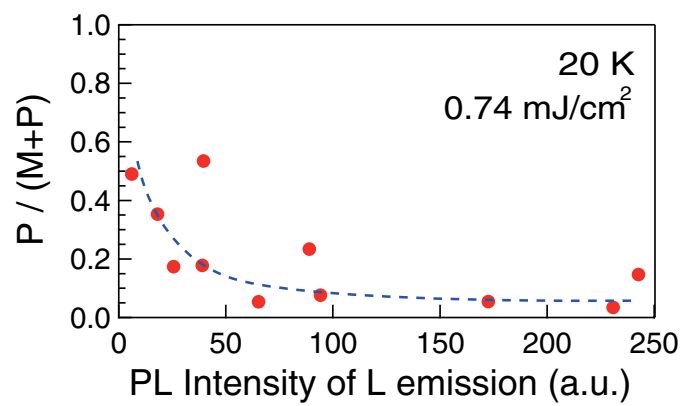

FIG. 7. (Color online) PL intensity of the $P$ emission relative to that of the $M$ plus $P$ emissions as a function of the integrated intensity of the $L$ emission under the same excitation photon density of $0.74 \mathrm{~mJ} / \mathrm{cm}^{2}$. The dashed line is a visual guide.
$0.74 \mathrm{~mJ} / \mathrm{cm}^{2}$. Figure 7 shows the intensity of the $P$ emission relative to that of the sum of the $P$ and $M$ emissions $(P / P+M)$ as a function of the PL intensity of the $L$ emission. Here, the PL intensity of the $L$ emission was estimated from the spectral integration of the broad $L$ emission band. Given that the $L$ emission comes from a localized exciton trapped by potential fluctuations, the intensity of the $L$ emission depends on locations where the PL is detected. As seen in Fig. 7, the intensity of the $P$ emission clearly decreases as that of the $L$ emission increases, implying that the $P$ emission due to the exciton-exciton scattering process preferentially occurs at an area without potential fluctuations, whereas the $M$ emission due to the biexciton occurs even at the areas where potential fluctuations exist.

In $\mathrm{GaN}$ thin films, the four-wave mixing signal due to the biexciton-exciton transition $\left(X X_{A}\right)$ becomes strong along line defects relative to that due to the $A$-exciton transition $\left(X_{A}\right)$, suggesting that the exciton-exciton interactions are spatially modified by the defects. ${ }^{25}$ In InGaN alloy compounds, the PL due to the exciton-exciton scattering process preferentially occurs at places where spatial fluctuations of alloy composition exist, ${ }^{26}$ although this result is somewhat opposite to the result we observed here. Judging from these experimental results, we tentatively deduce that the excitonic and biexcitonic systems are separately formed and relaxed in space in the layered-type $\mathrm{PbI}_{2}$ because of spatially distributed potential fluctuations located between layers. Further experiments such as microscopic time-resolved PL measurements are needed to confirm our conjecture.

\section{CONCLUSION}

In summary, we have investigated ultrafast PL dynamics due to biexcitons and exciton-exciton scattering in the layeredtype semiconductor $\mathrm{PbI}_{2}$ by using the $\mathrm{OKG}$ method. Under band-to-band excitation with intense excitation density, we simultaneously observed $P$ and $M$ emissions. We found that the $P$ emission appears with a time delay whose latency increases as the excitation photon energy increases. The latency to onset indicates that the $P$ emission occurs after the energy relaxation of excitons with an excess energy via the exciton-LO phonon scattering. In contrast, the $M$ emission appears instantaneously after the excitation and the spectrum has an asymmetric shape due to thermal distribution of biexcitons and biexciton-biexciton collisions. From the time-dependent peak energy shift of the $P$ emission and spectral shape analysis of the $M$ emission, we could evaluate the effective temperatures of the excitonic and biexcitonic systems. These experimental results strongly suggest that the $P$ and $M$ emissions occur separately in space with different relaxation processes; the former relaxing radiatively by inelastic scattering between excitons and the latter being annihilated via biexciton-biexciton and biexciton-exciton collisions.

\section{ACKNOWLEDGMENTS}

This work was supported in part by Grants-in-Aid for Scientific Research (Grants No. 23241034, No. 23104515, and No. 23104713) from JSPS and MEXT. 
${ }^{1}$ T. Nagai, A. Yamamoto, and Y. Kanemitsu, Phys. Rev. B 71, 121201 (2005).

${ }^{2}$ H-J. Ko, Y. F. Chen, T. Yao, K. Miyajima, A. Yamamoto, and T. Goto, Appl. Phys. Lett. 77, 537 (2000).

${ }^{3}$ Y. Kagotani, K. Miyajima, G. Oohata, S. Saito, M. Ashida, K. Edamatsu, and T. Itoh, J. Lumin. 112, 113 (2005).

${ }^{4}$ H. Ichida, Y. Kanematsu, K. Mizoguchi, D. Kim, and M. Nakayama, Phys. Rev. B 76, 085417 (2007).

${ }^{5}$ N. Arai, J. Takeda, H.-J. Ko, and T. Yao, J. Lumin. 119-120, 346 (2006).

${ }^{6}$ D. Fröhlich and R. Kenklies, Nuovo Cimento 38, 433 (1977).

${ }^{7}$ J. Takeda, T. Goto, M. Tomita, and M. Matsuoka, J. Phys. Soc. Jpn. 58, 1441 (1989).

${ }^{8}$ T. Makino, P. Gu, M. Watanabe, and T. Hayashi, Solid State Commun. 93, 983 (1995).

${ }^{9}$ K. Tanaka, T. Hosoya, R. Fukaya, and J. Takeda, J. Lumin. 122-123, 421 (2007); T. Hosoya, K. Tanaka, R. Fukaya, and J. Takeda, ibid. 119, 24 (2006).

${ }^{10}$ J. Takeda, T. Ishihara, and T. Goto, Solid State Commun. 56, 101 (1985).

${ }^{11}$ S. Mitsubori, I. Katayama, S. H. Lee, T. Yao, and J. Takeda, J. Phys.: Condens. Matter 21, 8064211 (2009).

${ }^{12}$ J. Takeda, K. Nakajima, S. Kurita, S. Tomimoto, S. Saito, and T. Suemoto, Phys. Rev. B 62, 10083 (2000); J. Lumin. 87-89, 927 (2000).

${ }^{13}$ M. Watanabe, T. Hayashi, and R. Kato, J. Phys. Soc. Jpn. 59, 4526 (1990).
${ }^{14}$ S. Shionoya, H. Saito, E. Hanamura, and O. Akimoto, Solid State Commun. 12, 223 (1973).

${ }^{15}$ C. Klingshirn, Phys. Status Solidi 71, 547 (1975).

${ }^{16} \mathrm{~A}$ numerical analysis using complicated rate equations including various kinds of collisions and relaxations might be possible to fit the decay profiles of the self-energy. However, here, we simply introduce a biexponential decay profile to fit the experimental results phenomenologically.

${ }^{17}$ J. Takeda, N. Arai, Y. Toshine, H. J. Ko, and T. Yao, Jpn. J. Appl. Phys. 45, 6961 (2006).

${ }^{18}$ T. Goto and Y. Nishina, Solid State Commun. 31, 369 (1979).

${ }^{19}$ G. Lucovsky, R. M. White, W. Y. Liang, R. Zallen, and Ph. Schmid, Solid State Commun. 18, 811 (1976).

${ }^{20} \mathrm{G}$. Oohata, Y. Yokotsuji, D. G. Kim, H. Ishihara, and M. Nakayama, J. Phys. Soc. Jpn. 78, 024702 (2009).

${ }^{21}$ S. Yamazaki and T. Goto, J. Phys. Soc. Jpn. 51, 3228 (1982).

${ }^{22}$ H. Akiyama, T. Kuga, M. Matsuoka, and M. Kuwata-Gonokami, Phys. Rev. B 42, 5621 (1990).

${ }^{23}$ M. Nakayama, S. Wakaiki, K. Mizoguchi, D. Kim, H. Ichida, and Y. Kanematsu, Phys. Status Solidi C 3, 3464 (2006).

${ }^{24}$ M. Ueta, H. Kanzaki, K. Kobayashi, Y. Toyozawa, and E. Hanamura, Excitonic Processes in Solids (Springer-Verlag, Berlin, 1986).

${ }^{25}$ Y. Toda, T. Ishiguro, K. Yamaguchi, K. Hoshino, K. Tadatomo, and S. Adachi, Phys. Status Solidi C 6, S684 (2009).

${ }^{26}$ M. Nakayama, R. Kitano, M. Ando, and T. Uemura, Appl. Phys. Lett. 87, 092106 (2005). 